\title{
Rigidity Percolation in Embryo Morphogenesis: Physics meets Biology (Again)
}

\author{
Rigidity percolation uncovers a structural basis for embryonic tissue phase \\ transitions \\ Authors: Nicoletta I Petridou, Bernat Corominas-Murtra, Carl-Philipp Heisenberg, \\ and Edouard Hannezo
}

Cell, 184(7):1914-1928, 2021

\begin{abstract}
Recommended with a Commentary by Sumit Sinha ${ }^{1}$ and D. Thirumalai ${ }^{2},{ }^{1}$ Department of Physics, ${ }^{2}$ Department of Chemistry, University of Texas at Austin, Austin, TX 78712, USA.
\end{abstract}

The material properties of embryonic tissues influence morphogenetic processes, such as body axis elongation and convergent extension. Advances in in-vivo experimental techniques have made it possible to decipher spatial organization and temporal dynamics, which have resulted in quantitative understanding of the impact of mechanical cues on cellular development $[1,2,3,4]$. In particular, direct measurements of viscosity, yield stress and bulk-modulus, have revealed that tissue development is accompanied by dynamic changes in material properties. Here, we highlight a couple of key findings in a recent spectacular study [4], which has revealed that zebrafish blastoderm organization can be described using the framework of phase transitions, thus providing a deep link between physics and biology. By combining direct imaging experiments, theoretical models of rigidity percolation (previously used to describe network glasses), the authors convincingly show that zebrafish blastoderm undergoes a genuine rigidity phase transition.

In non-living systems, phase transitions (PTs) are ubiquitous, and are accompanied by abrupt or continuous changes in macroscopic properties in response to smooth variations in control parameters. A familiar example is the first order phase transition between a liquid and a gas (for example liquid water to vapor formation) that is accompanied by a discontinuous change in the density (macroscopic variable) as the temperature (control parameter) is varied over a narrow range. Similarly, in the paramagnetic to ferromagnetic transition, which is a prototypical example of the second order PT, the magnetization (analogue of density) vanishes when the temperature approaches the Curie temperature. The finding that the blastoderm (a single layer of embryonic epithelial tissue) undergoes a PT [4] as the connectivity of the cells in the tissue reaches a critical value is a surprise, especially considering that the system is likely to be out of equilibrium.

The remarkable study [4] shows that zebrafish morphogenesis is characterized by the rigidity phase transition that has been well investigated in the context of central-force random 
networks in which the nodes are connected by Hookean springs with certain probability [5]. Although there are a number of intriguing results in the Petridou et al. study, here we focus on two key findings. (i) The analogue of the the blastoderm viscosity (macroscopic property), measured using micropipette aspiration techniques, suddenly decreases by an order of magnitude at the onset of morphogenesis. The abrupt decrease in viscosity $(\eta)$, indicative of fluidization, is followed by a slow thickening process $(\sim 1 \mathrm{hr})$ where the viscosity increases gradually. (2) To explain the changes in $\eta$ (see Figure 1C in [4]), they extracted cell connectivity by analyzing the confocal images and supplemented the analysis by performing numerical 2D simulations. The microscopic control parameter that regulates the PT is the cell-cell connectivity, which in turn depends on adhesive interaction strength between the cells. The combined analyses of experimental and simulations data showed that the giant cluster size increases abruptly as the normalized mean connectivity, $\langle k\rangle$, the control parameter that is physically motivated proxy for cell-cell adhesion, exceeds $\left\langle k_{c}\right\rangle \sim 2 / 3$. This finding demonstrated that the non-confluent (fractional coverage of cells is unity in confluent tissues but is less than unity in non-confluent tissues) blastoderm tissue undergoes a genuine PT, familiar in abiotic systems [5, 6, 7]. Quantitative analysis, using simulations that are informed by experiments, showed that PT has two characteristics. (a) The correlation length diverges as $\left\langle k_{c}\right\rangle$ is approached. (b) Power-law distribution of the cluster sizes at the critical point [8]. The cluster-size distribution as a function of the cluster size, other than the giant cluster, decays as a power law with an exponent around $\sim-2.5$.

The critical point $\left\langle k_{c}\right\rangle \sim 2 / 3$, signaling the onset of rigidity, is thought to be a second order PT, just like in the study by Jacobs and Thorpe [5]. A few points that the zebrafish blastoderm undergoes, what appears to be an equilibrium second order PT, could be made. The correlation length, $\xi \sim\left(\langle k\rangle-\left\langle k_{c}\right\rangle\right)^{-\nu}$ with $\nu=1$.16. Assuming that hyperscaling, $\alpha=$ $2-\nu d,(\alpha$ is the "specific-heat" critical exponent characterizing the divergence of the second derivative of the fraction of floppy modes near $\left\langle k_{c}\right\rangle$ [5], and $d$ is the space dimension) holds, we obtain $\alpha \approx-0.32$. This value is larger than $\alpha \approx-0.48$ found in the purely topological random network [9]. For a first order PT, the expected values of $\alpha$ and $\nu=\frac{1}{d}$ are 1 and $0.5(d=2)$, respectively [10]. The departure of these values from the ones inferred in the the zebrafish blastoderm further supports the claim that the transition is second order in nature. (2) The Petridou et al. study found that the distribution of the sizes, $s$ of the rigid (non-percolating) clusters, other than the GC, scales as $P(s) \sim s^{-\gamma}$ with $\gamma \sim 2.5$, a value that is larger than $\tau \sim 2.1$ found in rigidity transition in disk-like objects with attraction [6]. (Note that in standard percolation theory the algebraic decay of $P(s)$ is usually characterized by $\tau$. We follow the notation used in the Petridou et al. study and use $\gamma$, nominally used to characterize the divergence of susceptibility like quantity, which in percolation accounts for the divergence of the mean size of non-percolating clusters [7].) Using the relation, $\tau=\frac{d}{d_{f}}+1$, which is satisfied in the previous studies $[6,5]$ the fractal dimension $d_{f} \sim \frac{4}{3}$, a value that deviates from the ones reported elsewhere. The smaller value of $d_{f}$, compared to previous studies $\left(d_{f} \approx 1.8\right)$ is likely because in the Petridou et al. study the attractive cell-cell contacts have finite lifetimes, and hence could break and reform.

Dependence of $\eta$ on cell fraction: Because $\left\langle k_{c}\right\rangle$ is directly related to cell fraction (see figure $\mathrm{S} 1 \mathrm{H}$ in [4]), the dependence of $\eta$ on $\phi$, the area fraction (referred to as cell fraction in [4]) could be obtained. Figure 1 shows that $\eta$ increases dramatically ( $\approx 2$ orders of magnitude) 


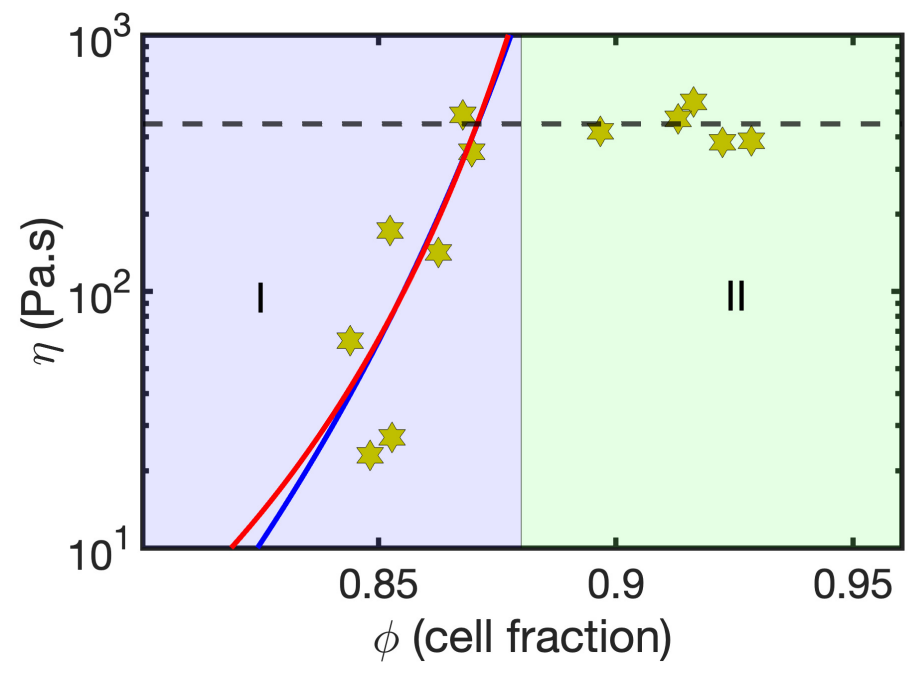

Figure 1: Fits of viscosity $(\eta)$ dependence on the cell area fraction $(\phi)$ using functional forms used to analyze glassy dynamics. The green hexagrams are experimental data points [4]. We fit $\eta$ in the interval $0.85 \leq \phi \leq 0.88$ (regime I) using Mode Coupling Theory $(\mathrm{MCT}), \eta \sim\left(1-\frac{\phi}{\phi_{M C T}}\right)^{-\gamma_{M C T}}$, in blue and the Vogel-Fulcher-Tamman (VFT) equation, $\eta \sim e^{\frac{D_{V F T} \phi}{\phi_{K}-\phi}}$, in red. The fit parameter $\phi_{M C T}=\phi_{K} \approx 0.95$. For $\phi \geq 0.88$ (regime II), $\eta$ saturates at $\sim 450$ Pa.s

over a narrow range of $0.85 \leq \phi \leq 0.88$ (regime I), and seems to saturate when $\phi \geq 0.88$ (regime 2). We analyzed the data in Figure 1 using theories for glassy dynamics. We fit $\eta(\phi)$ in region 1 using Mode Coupling Theory $(\mathrm{MCT})$ form $\left(\eta \approx\left(1-\frac{\phi}{\phi_{M C T}}\right)^{-\gamma_{M C T}}\right)$. The fit to the experimental data yields $\gamma_{M C T} \sim 3.7$, and $\phi_{M C T} \approx 0.95$. The value of $\gamma_{M C T}$ exceeds what is expected for hard sphere systems $[11,12]$ but is in line with particles interacting by soft potentials.

A hallmark of rigidity percolation is that the rearrangements in the rigid clusters require energy, and hence the dynamics should be activated. We found that a simple Arrhenius fit produces parameters that are non-physical. However, Vogel-Fulcher-Tamman (VFT) form $\left(\eta \approx \tau_{V F T} e^{\frac{D_{V F T} \phi}{\phi_{K}-\phi}}\right)$ accounts for the data with $\phi_{K}=\phi_{M C T}$. The parameter, $D_{V F T}$, which is a measure of fragility, is on the order of unity, a value that is considerably less than for hard sphere systems. But the zebrafish cells are soft, deformable objects, whose fragility like microgels, could be on the order of unity. In glassy systems, low fragility materials typically exhibit Arrhenius behavior for $\eta$ as a function of $\phi$ but this does not appear to be the case for zebrafish blastoderm. In this living system we encounter a strong glass $\left(D_{V F T} \approx 1\right)$ but with $\eta$ exhibiting VFT behavior, a characteristic of a fragile glass! It would be interesting to measure $\eta$ over a wider range of $\phi$, which may not be possible in living systems but would be achievable in simulations.

The saturation of $\eta$ for $\phi \geq 0.88$ (regime II) cannot be explained using the physics of glassy dynamics, as described above. However, similar behavior has been observed in 
soft colloidal systems [13], which has been attributed to some sort of non-equilibrium aging dynamics [13], possibly accompanied by reentrant fluidization. A finding in the soft colloid system is that the time dependence of the structure factor changes from stretched exponential $\left(\sim e^{-(t / \tau)^{\beta}}\right)$ with $\beta$ less than unity in regime I to compressed exponential $(\beta>1)$ in regime II). This prediction could be directly checked using simulation results in the Petridou et al. study [4].

Like all thought provoking studies, the results in the PT study of zebrafish blastoderm [4] raise questions that surely warrant additional scrutiny. From the perspective of physics, it is interesting to determine the universality class that could be ascribed to the rigidity phase transition. Although the Jacobs-Thorpe random rigidity percolation model is argued not to be in the universality class of connectivity percolation, relationships between the scaling

exponents, such as, $\beta=\nu\left(d-d_{f}\right)$ or $d_{f}=d-\frac{\beta}{\nu}$ are satisfied as can be checked using the reported values [5] of $\nu, \beta$ characterizing vanishing of the order parameter, and $d_{f}$. However, it has been suggested that particle systems (disks or spheres), at zero temperature, in which attraction is important [6,7] might be in a different universality class [6]. Our preliminary analysis of exponent relationship suggests that, although there is a genuine rigidity PT in zebrafish blastoderm, it does not seem to be covered by current models of rigidity percolation.

From the perspective of biology, it is unclear what advantages are conferred to an evolving system that is poised at criticality or close to it. By now it is well appreciated that on all length scales, ranging from nm scale exemplified by cooperativity in protein folding [14] to collective movement at the tissue level, and beyond are at the edge of criticality [15, 16]. The observation of phase transition in zebrafish blastoderm is in line with this observation, and it provides a great opportunity to flush out the link between critical behavior, and development. In the process the connection between physics and biology might be further strengthened.

Acknowledgments: We are grateful to Edouard Hannezo and Shura Grosberg for useful comments.

\section{References}

[1] Alessandro Mongera, Payam Rowghanian, Hannah J Gustafson, Elijah Shelton, David A Kealhofer, Emmet K Carn, Friedhelm Serwane, Adam A Lucio, James Giammona, and Otger Campàs. A fluid-to-solid jamming transition underlies vertebrate body axis elongation. Nature, 561(7723):401-405, 2018.

[2] Elias H Barriga, Kristian Franze, Guillaume Charras, and Roberto Mayor. Tissue stiffening coordinates morphogenesis by triggering collective cell migration in vivo. Nature, 554(7693):523-527, 2018.

[3] Nicoletta I Petridou, Silvia Grigolon, Guillaume Salbreux, Edouard Hannezo, and CarlPhilipp Heisenberg. Fluidization-mediated tissue spreading by mitotic cell rounding and non-canonical wnt signalling. Nature cell biology, 21(2):169-178, 2019.

[4] Nicoletta I Petridou, Bernat Corominas-Murtra, Carl-Philipp Heisenberg, and Edouard Hannezo. Rigidity percolation uncovers a structural basis for embryonic tissue phase transitions. Cell, 184(7):1914-1928, 2021. 
[5] DJ Jacobs and MF Thorpe. Generic rigidity percolation in two dimensions. Physical Review E, 53(4):3682, 1996.

[6] Gregg Lois, Jerzy Blawzdziewicz, and Corey S O’Hern. Jamming transition and new percolation universality classes in particulate systems with attraction. Physical review letters, 100(2):028001, 2008.

[7] Dion J Koeze and Brian P Tighe. Sticky matters: Jamming and rigid cluster statistics with attractive particle interactions. Physical review letters, 121(18):188002, 2018.

[8] Cyril Domb. Phase transitions and critical phenomena. Elsevier, 2000.

[9] Donald J Jacobs and Michael F Thorpe. Generic rigidity percolation: the pebble game. Physical review letters, 75(22):4051, 1995.

[10] Michael E Fisher and A Nihat Berker. Scaling for first-order phase transitions in thermodynamic and finite systems. Physical Review B, 26(5):2507, 1982.

[11] T.R Kirkpatrick. Mode-coupling theory of the glass transition. Phys. Rev. A, pages 939-944, 1985.

[12] S.P Das. Mode-coupling theory and the glass transition in supercooled liquids. Rev. Mod. Phys., 76:785-851, 2004.

[13] Adrian-Marie Philippe, Domenico Truzzolillo, Julian Galvan-Myoshi, Philippe Dieudonné-George, Véronique Trappe, Ludovic Berthier, and Luca Cipelletti. Glass transition of soft colloids. Physical Review E, 97(4):040601, 2018.

[14] Mai Suan Li, Dmitri K Klimov, and D Thirumalai. Finite size effects on thermal denaturation of globular proteins. Physical review letters, 93(26):268107, 2004.

[15] Thierry Mora and William Bialek. Are biological systems poised at criticality? Journal of Statistical Physics, 144(2):268-302, 2011.

[16] M. A. Munoz. Colloquium: criticality and dynamical scaling in living systems. Rev. Mod. Phys., 90:031001, 2018. 